\title{
The Blue Brain Project: calibrating the neocortical column
} Sean Hill*1,2, Rajnish Ranjan ${ }^{2}$, Srikanth Ramaswamy2, Shaul Druckman', Albert Gidon ${ }^{3}$, Jie $\mathrm{BaO}^{2}$, Imad Riachi², Felix Schürmann ${ }^{2}$ and Henry Markram²

\author{
Address: ${ }^{1}$ IBM T.J. Watson Research Center, Yorktown Heights, New York, USA, ${ }^{2}$ Blue Brain Project, École Polytechnique Fédérale de Lausanne, \\ Lausanne, Switzerland and ${ }^{3}$ Department of Neurobiology, Hebrew University, Jerusalem, Israel \\ Email: Sean Hill* - seh@zurich.ibm.com \\ * Corresponding author
}

from Sixteenth Annual Computational Neuroscience Meeting: CNS*2007

Toronto, Canada. 7-12 July 2007

Published: 6 July 2007

BMC Neuroscience 2007, 8(Suppl 2):PII0 doi:I0.1 I86/I47I-2202-8-S2-PII0

(C) 2007 Hill et al; licensee BioMed Central Ltd.

The Blue Brain Project is an attempt to reverse-engineer and model the neocortical column, to explore how it functions and to serve as a tool for neuroscientists and medical researchers. In order to achieve the goal of automatically fitting models to the latest data from clearly defined sources, a series of calibration steps have been developed. Each calibration step includes a physiological database, analysis technique and comparison to model data. All data is scored for completeness and quality. The aspects of the neocortical model for which calibration steps have been implemented are: the volume and composition of the column, ion channels, single cell electrical behavior, morphology repair and cloning, synaptic properties, short- and long-term plasticity, synaptic integration, polysynaptic loops, touch detection, structural and functional connectivity and emergent phenomena. The result of the calibration process is a score indicating the overall precision and quality of the fit. This system provides a means to identify those areas which require additional biological data as well as those areas where the model is biologically accurate or in need of refinement. The calibration process will continue to develop, as further biological details become known, and guide the refinement of the neocortical column model. 